\section{Laser-Assisted Removal of a Foreign Body from the Esophagus}

Endoscopic removal of foreign bodies from the esophagus is often easy. However, extracting sharp bones still causes technical difficulties. We report here on a case of an intraesophageal bone that was removed after it had been devided with Nd: YAG laser irradiation.

A 41-year-old man was admitted to hospital due to substernal chest and abdominal pain. The first symptoms had appeared after a meal of jugged rabbit; the pain increased with swallowing. The physical examination was normal. A chest radiography showed a slight left pleural effusion. Thoracic and abdominal CT scans revealed an increased density in the mediastinal fat, compatible with minimal inflammation. An endoscopic examination revealed a piece of bone, $30 \mathrm{~cm}$ from the incisor teeth. The two ends of the bone had penetrated the esophagus wall perpendicularly and caused large ulcerations (Figure 1). Removal of the bone was impossible, due to the risk of esophageal laceration. After it was irradiated with an Nd: YAG laser (700 J in five shots), the bone broke into two parts (Figure 2). It was then easily extracted using alligator forceps. An immediate control showed two puric ulcerations, facing each other. The patient was treated with antibiotics and parenteral nutrition; an oral diet was resumed after eight days. One month later, the patient was free of symptoms.

The most common intraesophageal foreign bodies removed in adults are meat bolus and bones. In most cases, the objects are located in the middle and lower esophageal segments (1). When an impact food bolus is found in the esophagus, exploration should be carried out with biplanar radiographs to determine whether there is any bone (2). Penetration of a sharp foreign body into the esophageal wall may result in complications such as perforation, vascular injury, and mediastinal infection (3). Fish and other bones therefore require extraction as soon as possible (3). At present the two recommended methods of decreasing the risk of perforation are the use of an overtube or a soft latex protector hood (4). When the sharp end of the foreign body is directed proximally, it can usually be grasped and carried into the stomach, where it can be turned, and then extracted without risk (1). However, these methods are not effective for extracting foreign bodies if both ends have entered the esophageal wall. Surgical removal is rarely indicated, except in this type of situation, or when complications have occurred (3). The present report is the first description of the extraction of an intraesophageal foreign body after initial cutting with an Nd: YAG laser. The procedure is apparently not traumatic, and can avoid laceration of the esophageal wall. A similar method was used by Kaltheuner et al. in 1990 to remove a foreign body in the colon (5).

When removal of a foreign body using conventional procedures appears impossible, therefore, cutting it with Nd: YAG laser irradiation may be attempted.

F.X. Weber: P. Deplaix. C. Barthélémy, P. Védrines, J. C. Audigier Dept. of Gastroenterology and Hepatology, Hôpital Nord, Centre Hospitalier Universitaire de Saint-Etienne, Saint-Etienne, France

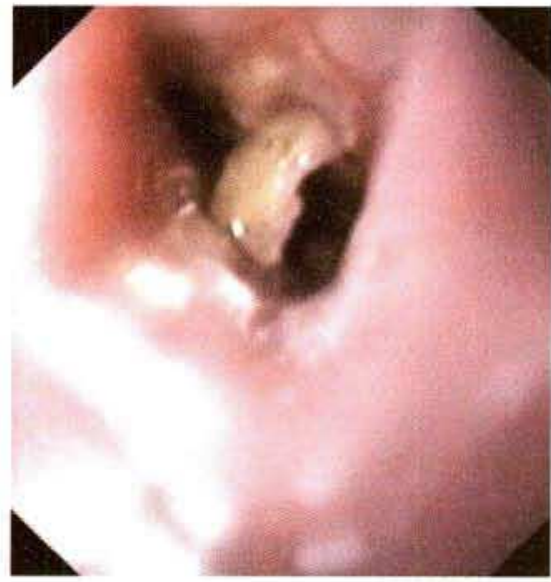

Figure 1: Endoscopic appearance of the impacted bone in the esophagus with two puric ulcerations facing each other.

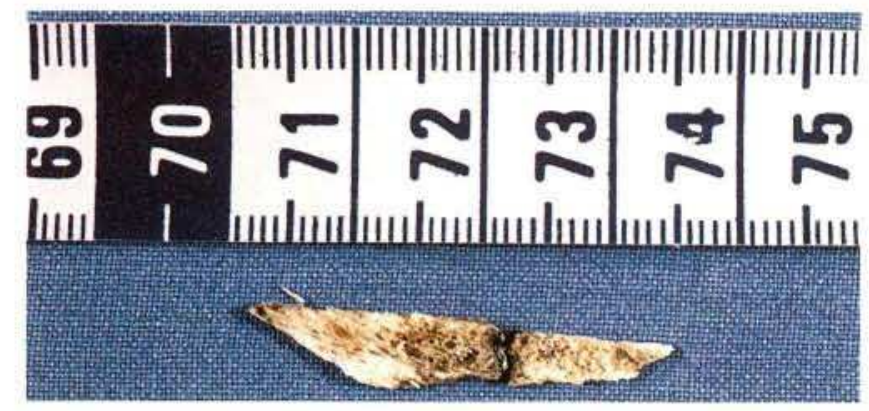

Figure 2: Appearance of the bone after laser cutting and removal.

\section{References}

1. Webb WA. Management of foreign bodies of the upper gastrointestinal tract: update. Gastointest Endosc 1995; 41: $39-51$.

2. Ginsberg GG. Management of ingested foreign objects and food bolus impactions. Gastrointest Endosc 1995: 41: 33 -8.

3. Brady PG. Esophageal foreign bodies. Gastroenterol Clin N Am 1991: 20: 691-701.

4. Bertoni G, Pacchione D, Conigliaro R, et al. Endoscopic protector hood for safe removal of sharp-pointed gastroesophageal foreign bodies. Surg Endosc 1992; 6: 255-8.

5. Kaltheuner M, Stallkamp H, Malchow H, et al. Laser-assisted removal of a foreign body from the colon. Surg Endosc 1992; 6: $78-9$.

Corresponding Author

C. Barthélémy, M. D.

Dept. of Gastroenterology and Hepatology

Hôpital Nord

Centre Hospitalier Universitaire de Saint-Etienne

42055 St-Etienne

France

Fax: $+33-7782-8452$ 